\title{
5th Bioanalysis in Clinical Research
}

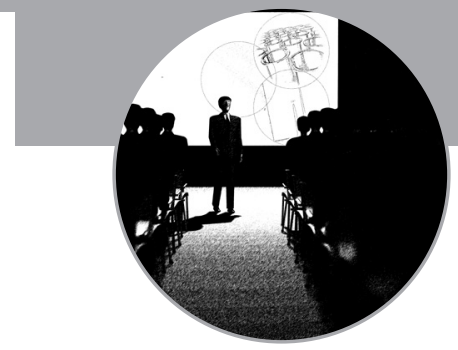

\section{The Conference Center at Victoria Plaza Hotel, London, UK, 23-26 February 2010}

The 5th Annual Bioanalysis in Clinical Research recently held in London, UK, covered a broad spectrum of interesting topics within bioanalytical method validation (BMV) including perspectives from the US FDA and European Medicines Agency (EMA) and implementation relating to method development and validation for small and large molecules. The theme of this year's meeting, "exploring the latest regulations and techniques in bioanalysis and learning how to practically implement them to improve your clinical trials", was evident throughout the sessions. The sessions consisted of a preconference workshop, 2 days of presentations along with group discussions and a postconference workshop. Topics included an overview of current EMA and US FDA bioanalysis validation guidelines, an approach to the validation of flow-cytometry methods in the regulatory environment, regulatory aspects in pre- and in-study BMV, regulated bioanalysis in support of clinical studies, regulations to follow in clinical laboratories (GLP and good clinical laboratory practice), crucial information on small-molecule drug metabolites, including metabolites in safety testing, validation of bioanalysis in clinical research, strategies for the analysis of therapeutic peptides in biofluids, the importance of transferring bioanalytical techniques (e.g., flow cytometry and multiplex bioanalysis) from the research laboratory to a regulated environment for use in early clinical trial end points, and development and validation of new technologies for faster analysis. The meeting attracted delegates from all over the world.

\section{Regulated bioanalysis}

Jeff Sailstad (Sailstad and Associates Inc., USA) presented his talk entitled 'Fit-for-purpose method validation for biomarker assays.' A practical, iterative, 'fit-for-purpose' approach to biomarker method development and validation was discussed, keeping in mind the intended use of the data and the intended regulatory requirements associated with that use. Particular attention should be paid to the application of research-grade diagnostic kits for biomarker assessments in support of clinical drug development.

Michael Zhou (Synta Pharmaceuticals Corporation, MA, USA) gave a presentation focusing on 'regulated bioanalysis in support of clinical trials.' Recently, the pharmaceutical industry has led global initiatives to develop a consensus regarding acceptable benchmark practices applicable to standardizing laboratory and management systems for bioanalytical studies supporting clinical trials. Recent issues with clinical bioanalytical support of drug-registration submissions demonstrate the additional need for a robust quality standard for bioanalysis of clinical samples. Industrywide acceptance and application of a new quality standard would assure the consistency and quality of data, applications and products approved for public use by regulatory agencies. In this presentation, historical background and current progress on regulated bioanalysis for clinical research was discussed. Ongoing activities regarding continuous improvement of quality from technical and regulatory perspectives were described using typical case studies.

'An approach to the validation of flow-cytometry methods in the regulatory environment' was given by Ruth Coldwell (AstraZeneca). This talk outlined an approach for the validation of flowcytometry methods used in the analysis of a wide range of biomarkers. It was presented as a guidance for method validation in a GLP environment and from the viewpoint of the pharmaceutical industry, but its relevance is wide ranging. The approach to method validation is intended as a starting point for further discussion, as well as providing reference material to colleagues developing fit-for-purpose flow-cytometry methods. Prevalidation steps were discussed as prerequisite assessments to determine method and reagent suitability, and minimize variables during the full validation process.

\section{Panelist discussion}

The panel discussion was led by Jeffrey Sailstad, along with panelists including Michael Zhou, Hendrik Neubert (Associate Research Fellow,

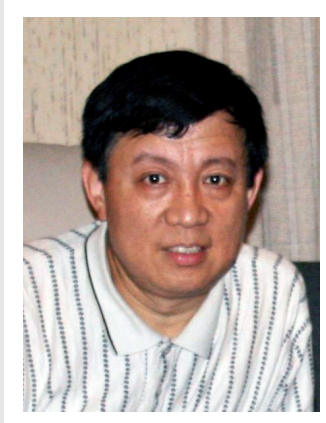

Michael Zhou, PhD

Director, Bioanalytical Chemistry/ DMPK, Synta Pharmaceuticals Corporation, 45 Hartwell Avenue, Lexington MA 0242I, USA Tel.: + I 781 54I 71।14 Fax: $+178|54| 6350$ E-mail: mzhou@syntapharma.com 
PDM Sandwich Laboratories, Biotherapeutics and Translational Research, Pfizer Inc., UK), and Berthold Lausecker (Head Development Bioavailability, F. Hoffmann-La Roche and Member of the Steering Committee, European Bioanalysis Forum [EBF], Belgium). This provided attendees with an overview of the applicable regulations for bioanalysis, with a focus on clinical studies. The overview truly connected the US FDA guidance on bioanalysis with the European Medicines Agency (EMA), International Conference on Harmonization (ICH), Organization for Economic Cooperation and Development (OECD) and other guidelines, and presented a practical translation of these guidelines towards bioanalytical activities. Panelists from Europe and the USA talked through their recent experiences and offered practical advice to delegates about how to comply with the latest regulations.

An update on the latest regulatory developments in bioanalysis method validation and study conduct was presented by Berthold Lausecker. This presentation covered some important topics, including:

- The current European regulations in bioanalytical method validation;

- The current European rules on study conduct;

- The challenges in meeting these requirements.

'Targeted LC-MS/MS quantification of protein biomarkers' was shared by Chris Barton (Bioanalytical Sciences, Quotient Bioresearch, UK). The discovery, quantification and validation of clinically relevant biomarkers require robust peptide- and protein-separation techniques. Rapid UPLC-MS/MS technologies have been applied to quantify a range of peptide and protein biomarkers in biological matrices, including both plasma and serum. The advantages of UPLC-MS/MS include (but are not limited to) shorter analysis times and improved reproducibility, making it suitable for use within a regulated environment. LC-MS/MS assays can then be developed to quantitatively analyze either intact proteins or unique peptides produced by enzymatic digestion of a target protein. Using such a method, the analysis and quantification of ten to 20 distinct proteins in a single 5-min analytical run is possible, with equivalent reproducibility to standard bioanalytical approaches. To expand the applicability of UPLC-MS/MS for the analysis of lower abundance protein biomarkers, targeted protein-enrichment techniques are required. The applicability of a number of different protein-extraction techniques and specific issues around validating LC-MS/MS was demonstrated using recent case studies performed at Quotient.

\section{Bioanalytical methodologies}

'A universal LC-MS approach for small molecules: application to the profiling of free fatty acids in human plasma', including the latest LC-MS technology, matrix effects and real-world application, was given by Professor Achille Cappiello (Laboratorio Cromatografia Liquida, Spettrometria di Massa, Dip. di Scienze Geologiche, Tecnologie Chimiche e Ambientali, Università degli Studi di Urbino, Italy). Although soft-ionization techniques represent the heart and soul of modern LC-MS, their performance is still impaired by a few crucial shortcomings that can be summarized as follows:

- Signal response is deeply influenced by mobilephase composition, polarity of analytes and is affected by interfering co-eluted substances (matrix effects);

- In small-molecule applications, the advantage brought by scarce fragmentation is paid for in terms of insufficient structural information, lack of reliable electronic libraries and a complex and costly MS instrumentation.

The development of an efficient LCMS interface based on a gas-phase, highenergy, electron ionization technique (LC-direct-EI-MS) overcomes most drawbacks and offers a more universal approach to the analysis of small molecules.

Ibtisam E Tothill (Cranfield University, UK) gave a presentation entitled 'Rapid biosensing techniques for point-of-care testing.' Advances in sensor technology have enabled the miniaturization of devices and multiplex testing of analytes as biomarkers in urine, blood/tissues and breath samples. It is believed that biosensor technology has the potential to use validated biomarkers for the development of pointof-care testing devices. Real-time sensor chips are being developed and validated for a range of applications such as cancer, cardiovascular disease and infectious micro-organisms. The introduction of nanomaterials in the developments of point-of-care devices has grown rapidly in recent years and this has resulted in the development of ultra-sensitive and rapid diagnostic platform for disease detection and 
diagnosis. This presentation gave an overview of the technology and the advances in the development of point-of-care testing devices.

\section{Best practices in metabolite quantitation}

This session was led by Philip Timmerman (European Head of Bioanalysis, Johnson \& Johnson, on behalf of the EBF, Belgium). It focused on the current industry practices in metabolite quantification. It also looked at what changes can be made to industry best practice in the future. The talk provided feedback on EBF discussions on:

- Metabolite quantification and metabolites in safety testing (MIST) or ICH M3(R2);

- Tiered approach versus validated methods;

- Qualified methods versus validated methods.

It covered progressive updates from EBF on their discussions on MIST in 2009, questionnaire in 2007, internal EBF discussions 2007-2008, results presented at the EBF Open Symposium in Barcelona (December 2008), Computer Validation Group, Montreal (April 2009) and APA Boston (September 2009). Results and discussion alluded that:

- Metabolite quantification is complex and follows multiple analytical strategies, which occur at all stages and with different levels of quality;

- With regards to increased metabolite quantification since MIST, it is unclear which quality standards are (should be) applied and there is some confusion about semantics (e.g., screening and semi-quantification). Thus, this provides an opportunity for optimization and harmonization. From the regulatory perspective, the final recommendation (White Paper) from the first quarter of 2010 focuses on three aspects: accordance with scientific criteria based on broad experience and CDER FDA 2001 (and CC-III enhancements); providing relief or alternatives where current processes do not align with specific study needs; understanding limitations of qualified assays and, finally, guiding appropriate use of resources in all phases of development.

At the end of this session, Philip Timmerman presented three proposed recommendations from the EBF perspective:

- Define three levels of quality for a 'tiered approach;'
- Provide clarification of the semantics of a 'tiered approach', focusing on qualified methods;

- Provide practical guidance on which quality standards to apply for MIST.

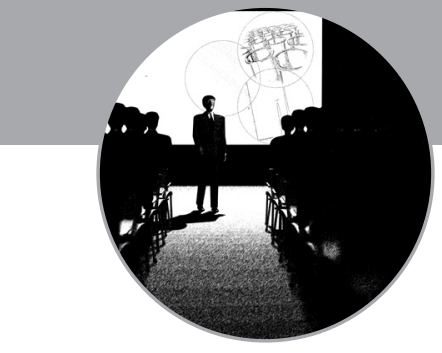

\section{White Paper update on ligand-binding assays}

'The assignment of statistically based confirmation cut points for immunogenicity testing: how to do it (and why one approach doesn't fit all)' was presented by Jeffrey Sailstad. In the most recent White Paper [1] from the Ligand Binding Bioanalytical Focus Group of AAPS, the intention was to put forth some of the best practices that have evolved since the original Mire-Sluis White Paper from 2004 [2]. In addition, the White Paper proposes approaches for the assignment of statistically based confirmatory cut points. As an industry, in a manner similar to what has been done for the screening cut point, Jeffrey Sailstad pointed out that we are in a similar position of 'test driving' and modeling the approaches proposed for the assignment of confirmation cut points. Undoubtedly, it is likely to encounter data sets that will challenge a standard approach to assigning a confirmation cut point. This presentation, by a bioanalyst who has the utmost respect for biostatisticians attempted to bridge the gap between the statistical numbers and the practical impact in the laboratory. The case study demonstrated what one might encounter in assigning the confirmation cut point based on inhibition of normal (adenosine deaminase negative) samples or, alternatively, assigning the confirmation cut point on the variability of low-level adenosine deaminase controls or possibly some hybrids to the two approaches.

\section{Group/delegate discussions}

The first day's interactive round-table discussion session and networking opportunity focused on 'real life examples of incurred sample analysis, repeat analysis: demonstrating that your assay is reproducible.' Delegates broke into small groups and worked through these questions for $20 \mathrm{~min}$. Then, each group nominated a spokesperson to report their group's conclusions back to the rest of the delegates. Delegates also discussed:

- What bioanalytical challenges are delegates experiencing at the moment?

- What practical solutions have been tried by other members of the group? 
The second day's interactive round table discussion was on 'assessing and implementing quality in bioanalytical studies: should GLP, good clinical laboratory practice (GCLP) or another system be followed?'

Delegates again broke into small groups and worked through these questions for $20 \mathrm{~min}$. Then, each group nominated a spokesperson to report their group's conclusions back to the rest of the delegates regarding the following:

- What are the GLP guidelines and is GLP suitable to follow?

- How do GCLP guidelines differ from GLP and will these be accepted as a standard?

- Using available texts to develop your own system of practice;

- How to ensure that all laboratories used are up to a suitable standard if they are not accredited.

\section{Conference workshops}

The conference also included a preconference workshop entitled 'New trends in LC and LC-MS' led by Professor Achille Cappiello. In recent years, different approaches have been taken to improve chromatographic performance in terms of analysis time and/or resolution. The use of columns packed with submicrometer particles in UPLC and the development of miniaturized separation and detection systems have widened the choice in many analytical applications. Furthermore, for the analysis of complex matrices (e.g., biological fluids, plant extracts, and food and environmental samples), MS or MS/MS interfacing provides a powerful analytical tool. Matrix effects are still a challenge in assessing accuracy and precision, representing a major drawback in quantitative analysis. The workshop covered emerging techniques in LC and LC-MS and their role in reducing matrix effects as follows:

- In which cases are LC-MS and LC-MS/MS most effectively used?

- How do matrix effects affect the accuracy and precision of the bioanalysis?

- How to evaluate matrix effects;

- Overcoming matrix effects in LC-MS;

- The role of emerging techniques.

In addition, a postconference workshop entitled 'GLP/regulated bioanalytical laboratories: technical and regulatory aspects from global perspectives' was given by Michael Zhou. This workshop provided attendees with a basic knowledge of GLP and bioanalytical laboratories by giving a historic background of GLP, its applicability and relationship to other regulations/principles, basic elements for GLP quality systems, case studies in executions - technical and regulatory aspects (e.g., current laboratory techniques and approaches for chromatographicbased methods [LC-MS/MS and ligand-binding assays]), and future trends from a global perspective. The workshop consisted of a broad scope of knowledge as follows:

- Introductory overview of essential roles of bioanalytical laboratories in pharmaceutical and biotechnological drug development;

- Fundamental understanding of regulatory aspects within laboratory operations: current and future requirements for GLP quality systems, facilities and personnel infrastructure and qualification along with continuing improvement on a daily basis;

- Strategies for sample preparation, along with essential concepts in extraction chemistry. Particular strategies for efficient use of automation within bioanalytical laboratories are also presented;

- The workshop concluded with information on recent advances in sample preparation, highthroughput procedures and analyses along with trends and initiatives on global regulations and implementations.

Attendees learned how to develop and implement strategies for routine, nonroutine and standard bioanalytical methods and applications in preclinical and clinical programs. It takes into account most international and national regulations, quality and accreditation standards such as GLP, cGMP, good clinical practice (GCP), GCLP and ISO 17025 worldwide, and corresponding interpretation and inspection guides.

\section{Summary}

This forum provided a broad scope of sessions regarding regulated bioanalysis for both small and large molecules in support of clinical research from a global standpoint. It certainly offered a good opportunity to stay informed and ahead on regulatory issues and technical challenges within bioanalysis. A future meeting will likely address top concerns and facilitate the implementation and execution of GLP, GCP and GCLP compliance. 


\section{Financial \& competing interests disclosure}

The author has no relevant affliations or financial involvement with any organization or entity with a financial interest in or financial conflict with the subject matter or materials discussed in the manuscript. This includes employment, consultancies, honoraria, stock ownership or options, expert testimony, grants or patents received or pending, or royalties.

No writing assistance was utilized in the production of this manuscript.

\section{Bibliography}

1 Shankar G, Devanarayan V, Amaravadi L et al. Recommendations for the validation of immunoassays used for detection of host antibodies against biotechnology products. J. Pharm. Biomed. Anal. 48(5), 1267-1281 (2008).

2 Mire-Sluis AR, Barrett YC, Devanarayan Y et al. Recommendations for the design and optimization of immunoassays used in the detection of host antibodies against biotechnology products. J. Immunol. Methods 289, 1-16 (2004). 